\title{
Die Geschichte des Flußnetzes in Norddeutschland und angrenzenden Gebieten
}

\author{
Von Paul Woldstedt, Bonn
}

Mit 1 Abbildung im Text und 1 Tafel (I)

Abstract. The river systems of North Germany show a characteristic alternating of peripheral („Urstromtäler") and centripetal ways („Durchbruchstäler"). The valley sections going to the north seem to be of especial importance. Mostly ancient river valleys, they were leading for the advancing ice, which conserved, and often deepened, them. In these channels dead ice was buried which, after melting, gave the rivers access to their ancient valleys.

Die norddeutschen ${ }^{1}$ ) Flüsse sind durchweg sehr jung. Bei vielen ist der heutige Verlauf überhaupt erst nach dem Verschwinden der letzten Eisdecke zustande gekommen. Die ältesten Flüsse Norddeutschlands stammen aus dem Jüngeren Tertiär, und zwar im wesentlichen erst aus dem Pliozän (vgl. für das folgende Taf. I).

Ein Paradestück in der Morphologie Norddeutschlands waren immer die Urstromtäler. Sie haben sich vor dem jeweiligen Eisrande entwickelt als echte Randtäler, in denen die Eisschmelzwässer und teilweise auch die von Süden kommenden Flüsse nach Westen zur Nordsee abflossen. Dabei waren die Urstromtäler nicht überall von vorneherein Flußtäler mit einheitlich nach Westen gehendem Gefälle; gelegentlich waren z. B. Staubecken eingeschaltet. Aber es fand im ganzen eine periphere Entwässerung zur Nordsee statt. Je älter die Urstromtäler sind, umso mehr sind sie durch spätere Vorgänge umgestaltet worden. Ausgeprägt ist das z. B. der Fall bei dem Breslau-Magdeburg-Bremer Tal, das zum Warthe-Stadium gehört. Heute ist ein einheitlicher Talboden nicht mehr vorhanden. Schwemmkegel der von Süden kommenden Mittelgebirgsflüsse auf der einen Seite, Flußdurchbrüche nach Norden zum nächsten Urstromtal auf der anderen Seite sowie noch weitere Vorgänge (wozu auch weitwellige Verbiegungen gehören können) haben das alte Randtal völlig verändert.

Das heutige ostelbische Flußnetz ist, wie ja seit langem bekannt, charakterisiert dadurch, daß bei den einzelnen größeren Flüssen jeweils Strecken in Urstromtälern abwechseln mit Durchbrüchen nach Norden. Am ausgeprägtesten ist das bei der Oder der Fall. Sie folgt von Breslau bis Parchwitz dem Breslau-Magdeburger Urstromtal, durchbricht dann das Warthe-Stadium, folgt dem Glogau-Baruther Urstromtal bis Neusalz, bricht nach Norden durch das Brandenburger Stadium hindurch, folgt dem Warschau-Berliner Tal bis Fürstenberg, durchbricht das Frankfurter Stadium, folgt dem Thorn-Eberswalder Urstromtal, um schließlich bei Oderberg in das Pommersche Stadium einzudringen. Ahnlich ist es bei dem größten Teile der übrigen Flïsse Norddeutschlands. Man könnte fast sagen: wo sich ihrem Drängen nach Norden ein Endmoränenzug entgegenstellt, da durchbrechen sie ihn.

Dies Durchbrechen in nördlicher Richtung ist ein so allgemeiner Zug, daß A. v. KoeneN (in mehreren Arbeiten, vgl. z. B. 1885) ihn nur tektonisch, d. h. durch rheinisch streichende junge Verwerfungen, erklären zu können glaubte. Heute kann gesagt werden, daß eine unmittelbare tektonische Beeinflussung dieser Durchbrüche keinesfalls in Frage kommt (daß aber vielleicht in einzelnen Fällen eine alte tektonische Anlage auf Umwegen doch eine Rolle spielen könnte). Für die meisten Durchbriiche war entscheidend das Heranreichen tiefer, toteiserfüllter Rinnen bis ins Endmoränengebiet bzw. sogar darüber hinaus. Durch die schematische Abb. 1 sei dies kurz erläutert.

1) Wenn im folgenden von Norddeutschland gesprochen wird, sind stets die angrenzenden Gebiete im Westen und Osten mitgemeint. 

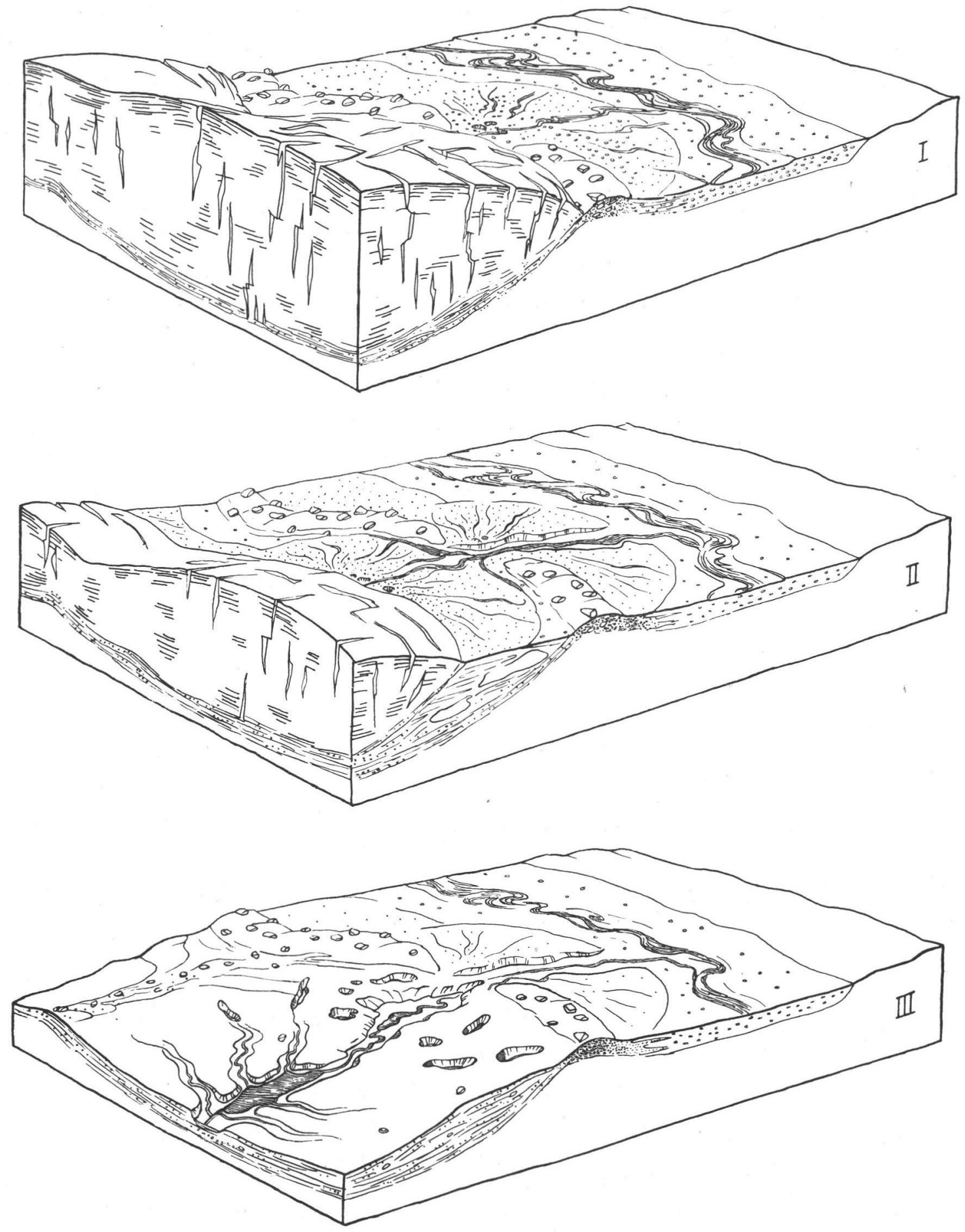


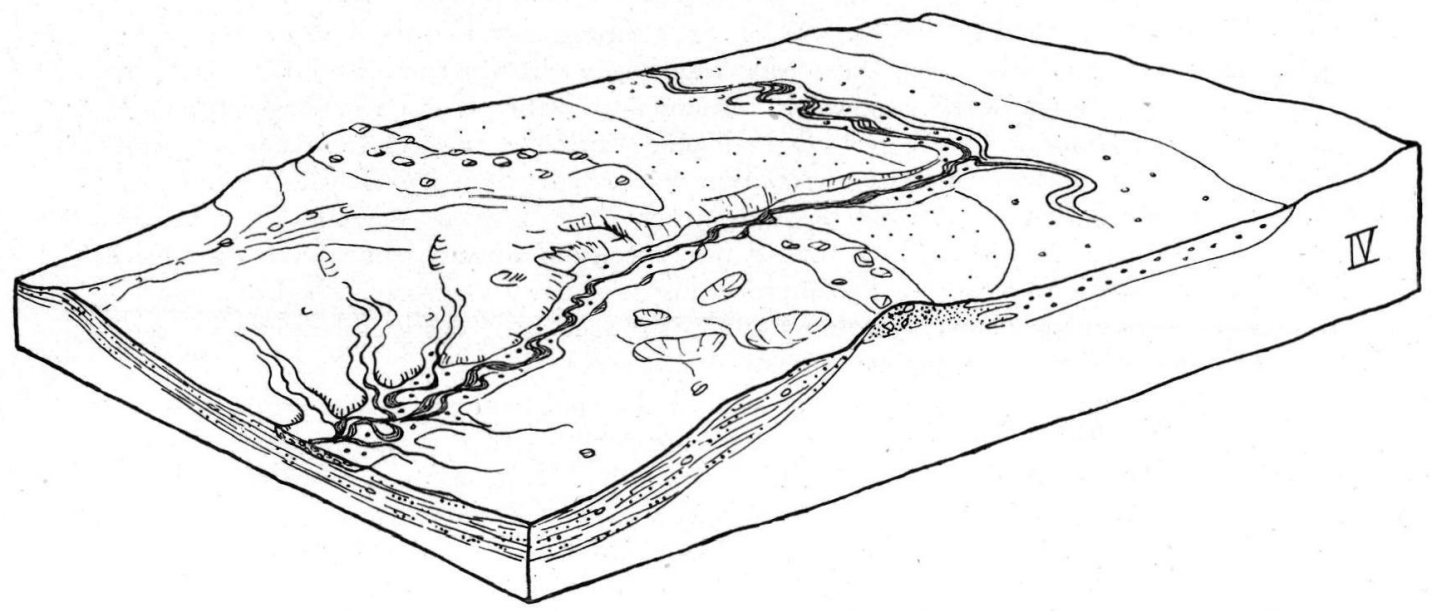

Abb. 1. Blockdiagramme, die Entstehung eines Durchbruchstals in zentripetaler Richtung verdeutlichend ${ }^{1}$ ).

In Phase I fließen die Schmelzwässer, durch eine Lücke der Endmoräne austretend, zum Urstrom ab, der von links nach rechts fließt. In Phase II ist der Eisrand zurückgegangen. An zahlreichen Stellen, besonders in der Mitte, ist Toteis im Untergrund liegengeblieben und übersandet worden. Die jetzt abfließenden Schmelzwässer schneiden sich in den Sanderkegel von Phase I ein und gelangen in den Urstrom.

In Phase III ist das Eis weiter zurückgegangen. Das versenkte Toteis schmilzt aus, besonders kräftig im mittleren Raum, wo sich teilweise auch Seen bilden und allmählich eine zentripetale Entwässerung entsteht. Von dem tiefliegenden Zweigbecken aus wird - Phase IV - in dem sandig-kiesigen Material sehr leicht der Urstrom angezapt und nun an die zentripetale Entwässerung angeschlossen.

In dieser Weise werden die meisten Flußdurchbrüche vor sich gegangen sein. Es gibt aber auch noch einfachere Fälle, wo sich unmittelbar vor dem Gletscher große Staubecken bildeten, die bei der Auflösung der Eisfront am tiefsten Punkte überliefen.

Entscheidend ist immer das Auftreten tiefer radialer Rinnen innerhalb der einzelnen Gletscherloben. Wie ich 1952 ausgeführt habe, handelte es sich hier jeweils um die am tiefsten in den Untergrund eingreifenden Partien des Gletschers. Dabei wurden die Rinnen, denen die vorrückenden Gletscher folgten, ausgestaltet durch das nachrückende Eis. Die Tiefenlinien aber, denen das Eis beim Vorrücken folgte, waren in vielen Fällen wiederum die alten, vorhandenen Flußtäler.

Es ist das eine Gesetzmäßigkeit, die immer wieder zu beobachten ist: die alten Tiefenlinien (Flußtäler u. dgl.) ziehen das Eis beim Vorrücken an. Sie bleiben beim Zurückgehen des Eises wiederum leicht erhalten, weil in ihnen das Eis am mächtigsten war und zuletzt austaute. Diese alten Flußlinien können natïlich, wie viele andere Flußtäler auch, ursprünglich tektonisch bedingt gewesen sein; und so kommt vielleicht bei einzelnen Flußdurchbrüchen in Norddeutschland eine ursprïngliche tektonische Anlage wieder zur Geltung, aber keinesfalls so, wie A. v. KoENEN und andere angenommen hatten, daß hier spät- oder postglaziale Verwerfungen eine Rolle gespielt hätten.

Aus dem Umstand, daß bei den meisten Flußdurchbrüchen die toteiserfüllten radialen Rinnen (Zweigbecken) eine Rolle spielten, ergibt sich das Alter der Flußdurchbrüche: sie traten ein, nachdem das in den Rinnen vorhandene Toteis teilweise oder gang ausgeschmolzen war. Die Durchbrüche im Bereich der Letzten Vereisung, d. h. vom Baruther Tal nach Norden hin, müssen in engem Anschluß an den Eisrückgang begonnen haben und waren spätestens mit Beginn der postglazialen Wärmezeit beendet.

1) Die Blockdiagramme wurden freundlicherweise von Herrn cand. geol. P. Wurster gezeichnet, dem ich dafür auch an dieser Stelle herzlich danken möchte. 
So liegt also der Gang der Entwässerung während der Letzten Eiszeit und in der Späteiszeit fest: randliche (periphere) Entwässerung nach Westen während der sich vorschiebenden Vereisung, während des Maximums und während der Rückzugsstadien. Da die sog. Rückzugsstadien zum großen Teil neue Vorstöße nach vorhergehenden Rückzügen darstellen, können in einzelnen Fällen inzwischen schon Durchbrüche weiter nach Norden stattgefunden haben, die bei den neuen Vorstößen wieder blockiert wurden. Auf die periphere Entwässerung während der Vereisung folgten sehr schnell die Durchbrüche nach Norden, die, vom Eiszentrum aus gesehen, als zentripetale Entwässerung bezeichnet werden können. Das Abwechseln peripherer und zentripetaler Strecken ist ja für den Verlauf der norddeutschen Flüsse so charakteristisch.

Es entsteht nun die Frage: wie war es in früheren Eiszeiten und Interglazialzeiten? Wir werden als das Wahrscheinlichste anzunehmen haben, daß es ähnlich war wie während der Letzten Eiszeit und der Postglazialzeit. Wir können das zunächst einmal für das Warthe-Stadium und die Letzte Interglazialzeit wahrscheinlich machen. Während des Warthe-Stadiums war das Breslau-Magdeburg-Bremer Urstromtal in Funktion, wahrscheinlich aber nur verhältnismäßig kurze Zeit, d. h. während des Maximums. Später waren sicher andere, weiter nördlich gelegene Urstromtäler in Funktion, die heute unter der Letzten Vereisung verborgen sind. Aber diese Urstromtäler gingen nicht durch das untere Elbetal zur Nordsee, sondern zunächst zur westlichen Ostsee. Wie sie dann weiter verliefen, wissen wir nicht. Eine durchgehende Talverbindung war jedenfalls nach H. IlliEs (1954) im Raum der unteren Elbe, besonders im Raum von Lauenburg noch nicht vorhanden.

Der Durchbruch der Elbe durch das Warthe-Stadium bei Magdeburg muß wohl in engem Anschluß an den Rückzug des Eises von diesem Stadium vor sich gegangen sein. Es liegen keinerlei Anzeichen dafür vor, daß die Elbe etwa in der Letzten Interglazialzeit noch durch das Ohre-Allertal nach Westen geflossen sei. Die Elbe muß damals in nördlichem Verlauf zur westlichen Ostsee gegangen sein. Ob ihr alter Lauf etwa über die Tiefenlinie Lewitzsenke-Schweriner See zur Wismarschen Bucht ging oder gar über die Warnow nach Rostock-Warnemünde, wo wahrscheinlich eine Bucht des Eem-Meeres vorhanden war, das entzieht sich einstweilen unserer Kenntnis, ist aber vielleicht einmal durch sedimentpetrologische Untersuchungen zu klären. Auf den Verlauf der anderen ostelbischen Flüsse in der Letzten Interglazialzeit kommen wir später zurück.

Die 1920 von mir beschriebenen Durchbrüche von Schtschara und Bug durch den Westrussischen Landrücken (die östliche Fortsetzung des Warthe-Stadiums) nüssen ebenfalls bald nach dem Rückzug des Eises vor sich gegangen sein.

Je weiter wir nun im Eiszeitalter zurückkommen, umso schwieriger wird es, die Dinge einwandfrei zu rekonstruieren. Aber doch finden wir einige Anhaltspunkte, so z. B. im westelbischen Gebiet. Außerordentlich wichtig ist der durch Geschiebe- und Schwermineral-Untersuchungen von C. G. MAarLeveld (1954) und R. D. Crommelin (1954) erbrachte Nachweis älterer Urströme, die östliches Material nach den Niederlanden brachten. Solche Urstrom-Ablagerungen sind sowohl für die Saale- wie für die Elster-Eiszeit nachgewiesen. Der Urstrom, den die vorrüickende Elster-Vereisung vor sich herschob, verfrachtete nach CROMMELIN's Untersuchungen zunächst besonders Kaolinsandmaterial. Später mengt sich mehr und mehr vom Mittelgebirge kommendes Material dazu, darunter einwandfrei solches aus dem oberen Elbegebiet. Daß auch bei den älteren Vereisungen die periphere Entwässerung nur ein vorübergehender Zustand war und durch zentripetale Durchbrüche in derselben Weise wie in der Spät-Weichseleiszeit abgelöst wurde, dafür können im folgenden einzelne Beispiele angeführt werden, wenn wir jetzt die einzelnen größeren Flüsse von Westen nach Osten kurz betrachten.

Der $\mathrm{R}$ he in ist durch die nordischen Vereisungen nur im Mündungsgebiet betroffen worden. Nur die Saalevereisung ist bis in sein Gebiet vorgedrungen. Aber er hat sich 
durch das Eis nur vorübergehend und unwesentlich aus seiner Bahn bringen lassen. Heute läuft er schräg durch die Saale-Endmoränenlandschaft hindurch.

Die Ems, offenbar schon vor der Saalevereisung vorhanden (vgl. W. HAACK 1935, H. WeHrLI 1941), fand anscheinend ihren alten Lauf nach dem Rückschmelzen des anscheinend nur kurzen Saale-Maximums sehr schnell wieder, und konnte bei Lingen, östlich des Bentheimer Lobus, durch das Rehburger Vorstoß-Stadium durchbrechen. Weiter nördlich ist der Lauf früher vielleicht weiter westlich am Fuße des Hondsrug entlang gegangen (H. LigTERINK 1954). Die Elstervereisung hatte die Urems höchstens im Unterlauf etwas ablenken können.

Eine interessante Geschichte hat die W e s e r. Sie hat sich, wie iiberhaupt das Flußnetz Mitteleuropas, im Pliozän entwickelt. Ihre Geschichte im Alt-Pleistozän ist noch nicht klar zu übersehen. Die Tatsache aber, daß die Porta Westfalica schon in der Holstein-Interglazialzeit etwa bis zu ihrer heutigen Tiefe eingeschnitten war, spricht dafür, daß die Weser diesen Einschnitt bereits im Alt-Pleistozän benutzt hat. Ein großer Fluß muß hier schon in altpleistozäner oder noch früherer Zeit eine erhebliche Erosionsarbeit geleistet haben. Dafür kommt eigentlich nur die Weser in Frage. Deutlicher übersehbar wird die Geschichte der Weser in der Elster-Saale-Interglazialzeit (Holstein-I.). Damals hatte die Weser bis zur Porta denselben Verlauf wie heute. Allerdings war das Durchbruchstal von Vlotho noch nicht vorhanden. Die Mittlere Terrasse geht unter dem PortaKame hindurch unmittelbar in nordwestlicher Richtung auf die Porta zu. Nördlich der Porta lief die Weser mit nordwestlicher Generalrichtung zur Nordsee, um in einer bisher unbekannten Gegend, aber sicher weit westlich von Bremerhaven, das Meer zu erreichen. Die Weser muß damals einen großen Schwemmkegel in Nordwestdeutschland aufgeschüttet haben. Wir finden ihre Ablagerungen in einer breiten Zone, die aus der Gegend von Uchte bis etwa Quakenbrück reicht (vgl. hierzu u. a. J. STOLlen 1923).

Dann wurde die Weser durch den ersten großen Vorstoß des Saaleeises, der bis zum Rehburger Stadium ging, nach Westen abgelenkt. Sie floß zwischen Wiehengebirge und Rehburger Stadium nach Westen. Ihre Ablagerungen sind bis in die Gegend von Bramsche nachgewiesen. Beim weiteren Vorrücken des Saaleeises wurden immer größere Teile des Weserlaufes beeinflußt. Es schob sich eine Eiszunge durch die Porta hindurch und staute die Weser zunächst bis in die Gegend von Hameln auf. Das Wasser fand vielleicht zunächst noch einen Abfluß - z. T. vielleicht sogar randlich über das Eis - nach Westen, so wohl vorübergehend auch durch das Längstal Bünde-Osnabrück. Wesermaterial ist dort allerdings nicht nachzuweisen (vgl. W. Dienemann 1942). Aber einem aus einem Staubecken überlaufenden Wasser kann man seinen Ursprung ja nicht ansehen. Bei weiterem Vorrücken wurde der Stau noch weiter flußauf wärts verlegt.

Das Saale-Maximum war offenbar, wie oben schon gesagt wurde, nur ein sehr kurzes Ereignis, und sehr schnell wurde zunächst die gesamte Eismasse bis zum Nordrand des Weser-Wiehengebirges tot, bald darauf aber auch im nördlichen Flachland. Nun fand die Weser ohne weiteres wieder das Loch der Porta, durch das sie ins Norddeutsche Flachland hinausschlüpfen konnte. Der direkte Weg, den sie zur Bildungszeit der Mittleren Terrassen-Schotter genommen hatte, war ihr durch die mächtige Aufschüttung des Porta-Kames verschlossen. Aber ein kleiner Umweg über Vlotho führte sie doch zum Ziel. Auch hier ist der Durchbruch offenbar sehr schnell erfolgt, und zwar im unmittelbaren Anschluß an das Zurückgehen bzw. Totwerden des Eises. Jetzt fand die Weser ihren Weg nach Norden zu ihrer heutigen Mündung bei Bremerhaven. Dieser Verlauf konnte auch nicht mehr beeinflußt werden durch die letzten großen Vorstöße des Saale-Eises, den Lamstedter Vorstoß und den des eigentlichen Warthe-Stadiums. Der Eisrand blieb überall nordöstlich des Wesertales, und dieses diente damals als Urstromtal (H. Illies 1952). 
Wir übergehen Leine und Oker, die eine verhältnismäßig einfache Geschichte hatten, und kommen zum System der E lbe, das wir oben bereits kurz behandelt haben. Der Oberlauf der Elbe im Böhmischen Becken erfuhr seine Ausgestaltung im Jungtertiär, die wesentliche Anderungen nicht mehr erlitt. Auch der Durchbruch durch das Elbsandsteingebirge ist präglazial (R. Grahmann 1933). Wie der genannte Verfasser weiter ausführt, ist die präglaziale Elbe („E-Talboden“) in nördlicher Richtung bis übei Klotzsche zu verfolgen. Der weitere Verlauf ist unbekannt. Die in das Durchbruchstal bis etwa Schandau vordringende Elstervereisung führte, wie GraHMANN gezeigt hat, nur zu einem kürzeren Aufstau der Elbewässer. Über den Verlauf der Elbe in der ElsterSaale-Interglazialzeit lassen sich einige Angaben machen. Es sind keine Anzeichen dafür vorhanden, daß sie bereits dem heutigen Lauf über Magdeburg gefolgt und etwa durch das Ohretal nach Westen geflossen sei. Das letztere war offenbar noch gar nicht da. Denn die Beberbach-Schotter (mit dem Paläolithikum von Hundisburg) setzen nach F. WIEgERs (1929) quer über das Tal nach Norden fort. Bei der Elbe haben wir einen von Dresden nach Norden gerichteten Lauf zu erwarten. Die Untersuchungen von K. Genieser (1955) im Gebiet von Senftenberg-Dobrilugk, von Schüller \& Müller (1937), REICHE (1937) und anderen für das Gebiet von Wietstock südlich Berlin machen einen Verlauf der Elbe in nördlicher Richtung bis in die Berliner Gegend wahrscheinlich. Wie die Elbe dann weiter verlief, d. h. ob sie etwa die bis in die Gegend von Dannenberg reichende Bucht der Holstein-See oder den in der Ostsee sich bis mindestens in die Gegend von Rügen erstreckenden Ausläufer des Holstein-Meeres erreichte, ist einstweilen ganz unbestimmt.

Weiter westlich müssen Saale- und Muldesystem bereits vorhanden gewesen sein. Sie sind wahrscheinlich dem heutigen Elbtal über Magdeburg gefolgt. Vielleicht gehören die Paludinen-Ablagerungen der Altmark bei Stendal diesem System an, das dann wahrscheinlich in die eben erwähnte Dannenberger Bucht des Holstein-Meeres mündete. Entscheidende Veränderungen im Mittel- und Unterlauf bewirkte dann, wie wir schon sahen, das Warthestadium. Zwar gelang der Elbe der Durchbruch durch das Warthestadium bei Burg, aber nicht gelang ihr der Durchbruch durch die neugeschaffenen, die alte Holstein-Bucht querenden großen Stauchendmoränen desselben Stadiums, die von der östlichen Lüneburger Heide zur Lauenburger Gegend verliefen. Sie muß dann, wie schon gesagt wurde, einen Abfluß nach Norden gefunden haben, der sie vielleicht in die westliche Ostsee (Eem-Meer) führte ${ }^{2}$ ). Den Durchbruch der Elbe bei Lauenburg nach Westen zur Nordsee erzwangen nach H. IlliEs (1952, 1954) erst die gesammelten Wässer Ostelbiens beim Vorrücken oder Maximum der Weichselvereisung.

Wir übergehen die kleineren Mittelgebirgsflüsse (Spree, Neiße, Bober), die genau wie die großen Ströme die Endmoränenzüge des Warthe- und Brandenburger Stadiums meist gradlinig durchbrechen, und kommen zur Oder. Ihre Geschichte zu rekonstruieren, ist recht schwierig. Von allen Vereisungen wurde sie besonders stark betroffen; ja die Elstervereisung, die fast bis zu ihren Quellen vordrang, brachte sie für kurze Zeit fast völlig zum Verschwinden. Ihre präglazialen Schotter sind nach P. AssmanN (1934) in nördlicher Richtung bis in die Gegend von Oppeln zu verfolgen. Aber über den weiteren Verlauf nach Norden sowohl in der Präglazialzeit wie in der ElsterSaale-Interglazialzeit tappen wir völlig im Dunkeln. In der nördlichen Fortsetzung des oberen Odertals liegt das nach den Untersuchungen von B. KRYGowsKI (1952) sehr alt angelegte Prosnatal. Ob es etwas mit der Oder zu tun hat, müßte untersucht werden. Wahrscheinlich erreichte die Oder damals, wenn auch möglicherweise auf etwas anderem Wege als heute, in der Gegend nördlich Stettin das Holstein-Meer. In der Letzten In-

2) Oder war damals die südwestliche Ostsee Land und ging die letztinterglaziale Elbe durch dies Gebiet und Holstein zur Eiderbucht (Nordmann-Rinne) des Eem-Meeres? Diese langgestreckte Bucht erinnert an ein Flußästuar, und man sollte einmal mit sediment-petrologischen Untersuchungen dieser Frage nachgehen. 
terglazialzeit wird der Oberlauf der Oder bis in die Gegend von Neusalz derselbe gewesen sein wie heute. Die Küste des Eem-Meeres haben wir in Pommern wohl etwa in der Gegend der heutigen Küstenlinie anzunchmen. Damit bildete sie aber nördlich Stettin eine Bucht, die anziehend auf die Gewässer zwischen Elbe und Weichsel wirken mußte. So ist es möglich, daß die Oder schon damals etwa in diesem Gebiet in das Eem-Meer mündete.

Wir kommen schließlich zur We i c h s 1. Ihre Geschichte liegt wieder etwas klarer zutage. Freilich wurde auch sie durch die Elstervereisung so gut wie ganz außer Funktion gesetzt, so daß wir über die präglaziale Weichsel wenig wissen. Aber dann muß der Fluß seinen heutigen Lauf mindestens bis in die Gegend von Pulawy entwickelt haben. Ja wahrscheinlich können wir ihn auch damals schon in der Warschauer Gegend vermuten. Dafür sprechen die in der dortigen Gegend unter der Saale-Grundmoräne weit verbreiteten Paludinen-Schichten. $\mathrm{Ob}$ wir damit auch die Paludinen-Schichten des unteren Weichselgebietes (z. B. Plutowo, Succase) in Zusammenhang bringen dürfen, ist natürlich sehr zweifelhaft, wenn auch vielleicht nicht unwahrscheinlich. Sie könnten aber auch zu ganz anderen Flüssen gehören.

Die Saale-Vereisung drang in einem Lobus oberhalb von Pulawy im Weichseltal nach Süden - ein Beweis, daß hier ein Tal vorhanden war. Die W'eichselwässer wurden vor dem Eise gestaut. Ein zweites Mal wurden sie aufgestaut beim Warthe-Vorstoß, wovon Bändertone zwischen Warka und Demblin zeugen. Aber der Durchbruch durch das Warthe-Stadium gelang dann anscheinend sehr schnell, und in der Letzten Interglazialzeit hatte die Weichsel offenbar schon einen ganz ähnlichen Verlauf wie heute. Eine Bucht des Eem-Meeres erfüllte das Tal der unteren Weichsel. Nach G. MAAs (1904) ging sie sogar bis Thorn und Argenau. Dieser ausgeprägten Tiefenlinie folgte wieder die Letzte Vereisung. Das ist besonders deutlich bei dem Frankfurter Stadium mit seinem bis Gombin und Plock vorgreifenden Lobus, ähnlich bei dem Neuenburger Lobus des Pommerschen Stadiums.

Nach dem Aufstau der Wässer im Warschauer Becken während des Brandenburger und Frankfurter Stadiums - bei teilweisem Abfluß durch das Bzuratal nach Westen fand sehr bald der übliche Durchbruch nach Nordwesten statt, schließlich, nach der Phase des Thorn-Eberswalder Urstromtals, der Durchbruch durch die verschiedenen Phasen des Pommerschen Stadiums.

$\mathrm{Zus}$ ammenf assung. Das Flußnetz von Norddeutschland ist seit der ElsterVereisung charakterisiert durch ein Abwechseln peripherer und zentripetaler Laufstrecken. Als ein besonders wichtiges Element erweisen sich die nach Norden gerichteten Talabschnitte, die offenbar im wesentlichen der ursprünglichen Abdachungsrichtung entsprechen. Sie treten immer wieder in Erscheinung. Der einzelne Fluß findet nicht immer sein eigentliches altes, südnördlich gerichtetes $\mathrm{T}$ al wieder, sondern oft auch ein anderes, paralleles. Aber immer erweisen sich die alten südnördlichen Linien als wichtig. Sie konnten es nur bleiben, weil auch das Eis bei seinem Vorrücken diesen Tiefenlinien folgte, sie konservierte und oft noch vertiefte. In den Rinnen blieb tief versenktes Toteis liegen, dessen Austauen dann den Flüssen den Zugang zu ihren alten Tälern wieder freimachte.

In den obigen Ausführungen ist die Geschichte des norddeutschen Flußnetzes nur in großen Zügen skizziert worden. Vieles ist noch unsicher; manches mag sich bei genauerer Untersuchung als ganz anders herausstellen. Aber hier ist ein weites und besonders lohnendes Gebiet für geschiebekundliche und sedimentpetrologische Untersuchungen vorhanden. Das gilt ganz besonders für die weitere Untersuchung von Bohrungen im Norddeutschen Flachlande. Aus meiner eigenen langjährigen Praxis auf diesem Gebiete weiß ich, daß bei der Durchsicht der Bohrproben auf die südlichen Geschiebe früher viel zu wenig geachtet worden ist. In den Bohrregistern erscheint dann bestenfalls die Bezeich- 
nung: „Einheimische Geschiebe“. Aber worum es sich im einzelnen handelt, das wird kaum je mitgeteilt. Schwermineralanalytische Untersuchungen wurden damals noch nicht ausgeführt. Sie werden es, zusammen mit einer eingehenden Erforschung der südlichen Geschiebe, in Zukunft ermöglichen, den Lauf der Flüsse in früheren Abschnitten des Pleistozäns weitgehend aufzuhellen, was übrigens wegen der Kiesvorkommen und wegen der Wasserführung auch von einiger praktischer Bedeutung sein kann.

Auf dieses aussichtsreiche Gebiet geschiebekundlicher und sedimentpetrologischer Untersuchung hinzuweisen, war ein wichtiger Anlaß dieser Ausführungen.

\section{$\mathrm{Schriften-Nachweis}$}

Assmann, P.: Zur Frage der Terrassenbildung an der oberen Oder. - Der Oberschlesier 1934, S. $137-147$.

Сrommelin, R. D.: Über den Einfluß der nord- und mitteldeutschen Flüsse auf das ältere Pleistozän der Niederlande. - Mitt. geol. Staatsinstitut Hamburg 23, S. 86-97. 1954.

Dienemann, W.: Über vermeintliche Weserablagerungen im Längstal Porta-Osnabrück. - Jb. Reichsstelle f. Bodenforsch. 62, S. 1-30, Berlin 1942.

Genieser, K.: Ehemalige Elbeläufe in der Lausitz. - Geologie 4, S. 223-279. Berlin 1955.

Grahmann, R.: Die Geschichte des Elbtals von Leitmeritz bis zu seinem. Eintritt in das norddeutsche Flachland. - Mitt. Ver. Erdkunde Dresden, N.F. 1933.

HaAcK, W.: Erläut. zur Geol. Karte von Preußen u.s.w., Lief. 336. Bl. Lengerich. - Berlin 1935.

IlliEs, H.: Die eiszeitliche Fluß- und Formengeschichte des Unterelbe-Gebietes. - Geol. Jb. 66, S. 525-558. Hannover 1952. - - Entstehung und eiszeitliche Geschichte der unteren Elbe. - Mitt. geol. Staatsinstitut Hamburg 23, S. 42-49. 1954.

Koenen, A. von: Über Dislokationen westlich und südwestlich vom Harz. - Jb. preuß. geol. Landesanst. f. 1884, S. 44-55. Berlin 1885.

Ligterink, G. H.: De Hondsrug en het dal van de Oer-Ems. - Tijdschr. K. nederl. aardrijsk. Gen. \%1, S. 105-121. 1954.

MaArleveld, G. C.: Über fluviatile Kiese in Nordwestdeutschland. - Eiszeitalter u. Gegenwart 4/5, S. 10-17. 1954.

MAAs, G.: Uber präglaziale marine Ablagerungen im östlichen Deutschland. - Z. deutsch. geol. Ges. 56, Briefl. Mitt. S. 21-24. 1904.

Reiche, E.: Die Bedeutung südlicher Gesteine im Bereich des jüngeren Diluviums. - Z. f. Ge-schiebeforsch. 13, S. 76-86. Leipzig 1937.

Schüller, A. \& MülLER, H.: Über Geschiebe südlicher Herkunft aus der Umgebung von Berlin. - Z. f. Geschiebeforsch. 13, S. 28-39. Leipzig 1937.

Stoller, J.: Beiträge zur Geologie der ehemaligen Grafschaften Hoya und Diepholz I. - Jber. niedersächs. geol. Ver. 16, S. 1-27. Hannover 1923.

WEHRLI, H.: Interglaziale und vor-saaleeiszeitliche Ablagerungen in der Münsterschen Bucht. Z. deutsch. geol. Ges. 93, S. 114-127. 1941.

Wiegens, F.: UUber Gliederung und Alter des Magdeburger Diluviums. - Jb. preuß. geol. L.Anst. 50, S. 29-124. Berlin 1929.

Woldstedt, P.: Die Durchbrüche von Schtschara und Bug durch den Westrussischen Landrücken. - Z. Ges. Erdkunde Berlin, S. 215-225. 1920. - - Die Entstehung der Seen in den ehemals vergletscherten Gebieten. - Eiszeitalter u. Gegenwart 2, S. 146-153. 1952.

Manuskr. eingeg. 15. 4. 1955.

Anschrift des Verf.: Prof. Dr. Paul Woldstedt, Bonn, Argelanderstr. 118. 